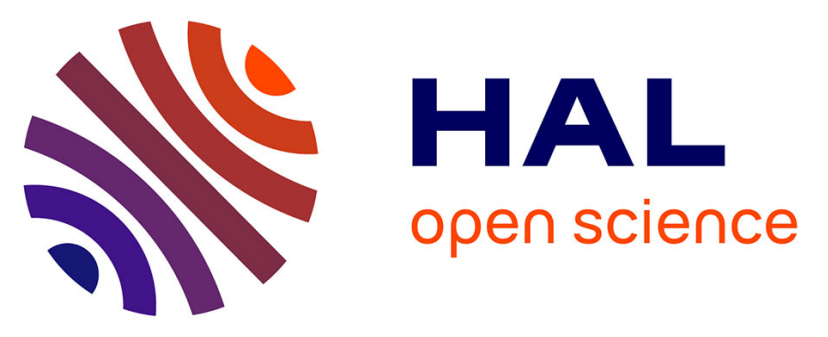

\title{
Photostability of single-walled carbon nanotubes/polymer core-shell hybrids as Telecom Wavelength Emitters
}

Lucile Orcin-Chaix, Stéphane Campidelli, Loïc Rondin, Frédéric Fossard, Fabien Bretenaker, Yannick Chassagneux, Christophe Voisin, Jean-sébastien Lauret

\section{To cite this version:}

Lucile Orcin-Chaix, Stéphane Campidelli, Loïc Rondin, Frédéric Fossard, Fabien Bretenaker, et al.. Photostability of single-walled carbon nanotubes/polymer core-shell hybrids as Telecom Wavelength Emitters. ACS Applied Nano Materials, 2020, 3, pp.7291-7296. 10.1021/acsanm.0c01732 . cea02890004

\section{HAL Id: cea-02890004 https://hal-cea.archives-ouvertes.fr/cea-02890004}

Submitted on 5 Oct 2020

HAL is a multi-disciplinary open access archive for the deposit and dissemination of scientific research documents, whether they are published or not. The documents may come from teaching and research institutions in France or abroad, or from public or private research centers.
L'archive ouverte pluridisciplinaire HAL, est destinée au dépôt et à la diffusion de documents scientifiques de niveau recherche, publiés ou non, émanant des établissements d'enseignement et de recherche français ou étrangers, des laboratoires publics ou privés. 


\title{
Photostability of single-walled carbon nanotubes/polymer core-shell hybrids as Telecom Wavelength Emitters
}

\author{
Lucile Orcin-Chaix, ${ }^{\dagger}$ Stéphane Campidelli, ${ }^{\ddagger}$ Loïc Rondin, ${ }^{\dagger}$ Fabien Bretenaker ${ }^{\dagger}$ \\ Yannick Chassagneux, "Christophe Voisin, "and Jean-Sébastien Lauret*,† \\ Université Paris Saclay, ENS Paris Saclay, Centrale Supelec, CNRS, LUMIN, 91405 \\ Orsay Cedex, France, France;, UniversitÂA' Paris-Saclay, CEA, CNRS, NIMBE, LICSEN, \\ 91191, Gif-sur-Yvette, France, and Laboratoire de Physique de l'Ecole Normale Supérieure, \\ ENS, PSL, CNRS, Université de Paris, Sorbonne Université, 75005 Paris, France. \\ E-mail: lauret@ens-paris-saclay.fr
}

\section{Abstract}

In the spirit of the core-shell approach that made it possible to greatly enhance the photostability of semiconductor nanoparticles, we study the emission stability of single carbon nanotubes emitting at telecommunication wavelengths protected by a double shell of polymer. Two types of structures with different polymer layers have been studied at cryogenic temperatures, whereas nanotubes wrapped with poly (9,9-di-n-octylfluorenyl-2,7diyl) and embedded in a bulk polystyrene matrix were used as a reference. A Mandel parameter description applied to the emission intensity and an Allan deviation analysis of the spectral diffusion show quantitatively that the polymer shells stabilize the emission of single carbon nanotubes by more than one order of magnitude in comparison with micelle wrapped nanotubes deposited on a $\mathrm{SiO}_{2}$ surface. This represent an additional step towards the use of single-walled carbon nanotubes as quantum emitters at telecommunication wavelengths.

\footnotetext{
*To whom correspondence should be addressed

${ }^{\dagger}$ LUMIN

$\ddagger_{\mathrm{CEA}}$

ๆLPENS
}

\section{Keywords}

Carbon nanotubes, Hybrid nanomaterials, Functionalization, Luminescence, Single emitter

\section{Introduction}

Photonics and quantum photonics using singlewalled carbon nanotubes (CNT) as building blocks are developing strongly. Carbon nanotubes can be used as light sources, optical modulators or detectors. ${ }^{1-10}$ Strategies based both on films or on single nanotubes are currently being developed depending on the application. In particular, single nanotubes are promising sources of single photons emitting in the telecommunication window. ${ }^{5,11,12}$ In this context, the emission stability of single nanotubes is a key issue towards their use in quantum devices. Indeed, nanotubes being made only of surface atoms their properties are very sensitive to their local environment. While this extreme sensitivity is an asset for sensing applications, ${ }^{13}$ in optics it gives rise to the broadening of the emission peaks and to the instability of the emission spectrum including the socalled spectral jitter and blinking phenomena. ${ }^{14}$ These two processes are detrimental to the use 
(a)

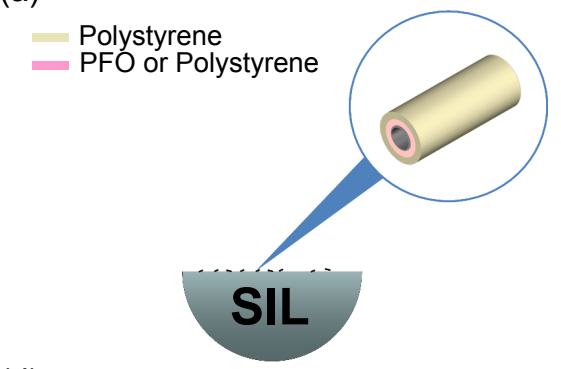

(d)

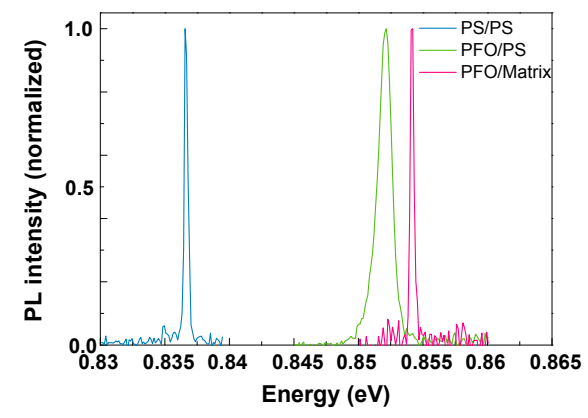

(b)

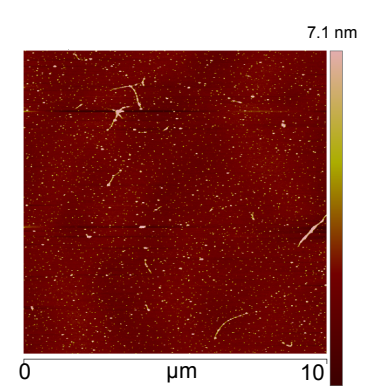

(e)

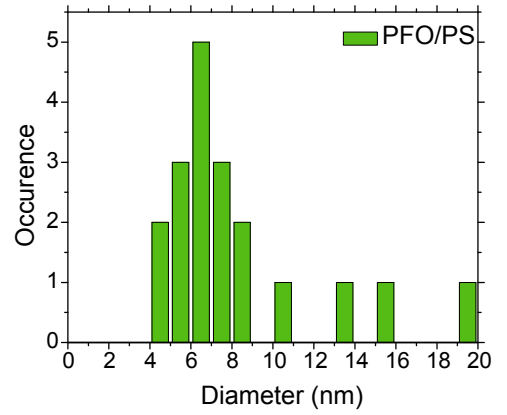

(c)

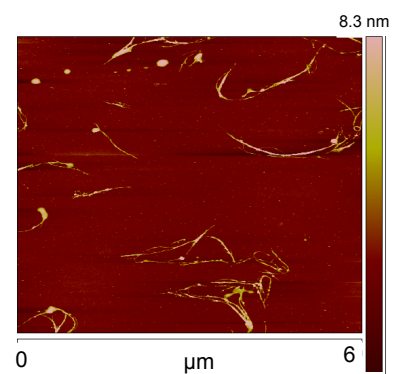

(f)

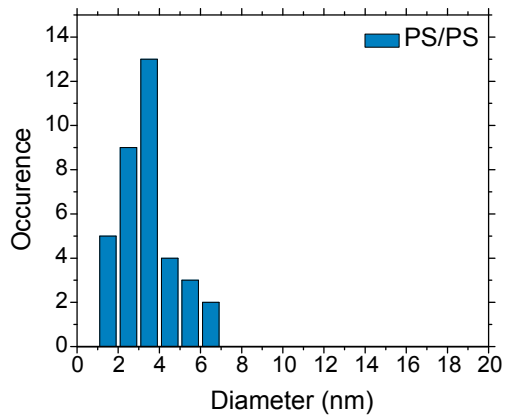

Figure 1: (a) Description of the samples deposited on the surface of a Solid Immersion Lens (SIL). (b)(c) Typical AFM images of CNT@PFO/PS and CNT@PS/PS core-shell structures respectively. (d) Typical PL spectra of single core-shell structures excited at $1.37 \mathrm{eV}$ (905 nm). Acquisition time:30s, excitation power: $\sim 150 \mu \mathrm{W}$. (e)(f) Histograms of heights of CNT@PFO/PS and CNT@PS/PS core-shell structures deduced from AFM measurements.

of nanotubes for application as single emitters for quantum technologies. In this context, the search for strategies improving the stability of the nanotubes' emission is an important question. For instance, it has been shown that embedding nanotubes in a polymer matrix can improve their optical performances. ${ }^{14,15}$ Nevertheless, once nanotubes are capped or embedded in a polymer layer, they can not be handled anymore. Recently, Noé et al showed that the emission of nanotubes deposited on an h-BN substrate is much more stable than when a $\mathrm{SiO}_{2}$ substrate is used. ${ }^{16}$ Nevertheless, in some cases, one would need to choose more common substrates such as $\mathrm{Si} / \mathrm{SiO}_{2}$ wafers in the view of building devices. Here, our strategy is to assemble core-shell structures where a polymer shell wraps the active core made of a CNT. This approach is inspired by the great improvement of the emission stability of semiconductor nanocrystals when they are capped with a protective shell. ${ }^{17,18}$ The objective is to combine the advantages of being able to choose any desired substrate and to perform postprocessing with a high emission stability.

Previously, we reported the synthesis of core-shell structures with HiPCo nanotubes. ${ }^{19}$ Briefly, a first layer of polymer is wrapped around the nanotubes through a non-covalent interaction. Non-covalent chemistry is used to prevent any damaging of the nanotube surface. The non-covalent layer showing poor stability, a second layer of polymer is build on the top of the first one by a radical polymerisation of polystyrene in order to lock the structure. These core-double-shell structures are very stable and can be solubilized in usual organic solvents making them easy to handle and process. ${ }^{19}$ The intended utility of the shell is both to protect the nanotube from being damaged during a device fabrication and to isolate the nanotube from its local environment to stabilize its emission.

In this paper, we study in particular the influence of the double shell structure on the luminescence stability of single nanotubes at cryogenic temperatures. Two types of structures have been synthesized. In addition 
to the combination of polystyrene and crosslinked polystyrene previously reported, ${ }^{19}$ a new combination of poly (9,9-di-n-octylfluorenyl-2,7diyl) (PFO) as inner shell with cross-linked polystyrene as outer shell has been synthesized. Moreover, in the view of using these structures as quantum emitters for quantum communications, the synthesis procedure has been applied to single-walled carbon nanotubes synthesized by laser ablation emitting in the $\mathrm{C}$ band of telecommunication. Furthermore, in order to benchmark these hybrids, we used PFO wrapped nanotubes in a bulk polystyrene matrix as a control sample. The stability of the luminescence intensity is analyzed using a Mandel parameter description and the spectral jitter is studied by means of the Allan deviation. Quantitative comparison is done with the control sample in the polystyrene matrix and with other reports in the literature. We find that the protective shells stabilize the emission by more than one order of magnitude in comparison with bare nanotubes deposited on a $\mathrm{SiO}_{2}$ surface ${ }^{16}$ and are nearly as good as the bulk polystyrene embedded CNTs, with the key advantage of remaining processable for their integration in devices. This demonstrates that the core-shell approach is promising towards the use of CNT as quantum emitters.

\section{Experimental}

Sample preparation. The nanotubes were synthesized by laser ablation. Their mean diameter is of $1.1 \mathrm{~nm}$, leading to an absorption peak at $\sim 1.55 \mu \mathrm{m} .{ }^{20,21}$ The synthesis of the core-double-shell structures has been reported previously ${ }^{19}$ and is described in the Supplementary information (SI). Here, the first shell non-covalently attached to the nanotube is composed either of PFO or polystyrene. The second layer used to lock the structure is made of crosslinked polystyrene. In the following, these two structures are respectively named CNT@PFO/PS and CNT@PS/PS. PFO has been chosen since it is a widely used polymer in nanotubes sorting, ${ }^{22}$ and in CNT based photonics devices. ${ }^{9,23}$ Moreover, polystyrene has proven its efficiency in stabilizing the luminescence of carbon nanotubes when used as a matrix. ${ }^{15}$ Finally, the combination of PFO nanotubes with a polystyrene matrix was shown to lead to a very stable emission of nanotubes at low temperature. ${ }^{1,24}$ Our approach tends to benefit from these advantages in an integrated system much simpler to handle experimentally. After the synthesis, the core-shell structures are dispersed in THF and then spin-coated on the flat surface of a solid immersion lens (SIL) to enhance the collection of photons in photoluminescence experiments (see figure 1(a)). Likewise, PFO wrapped nanotubes in toluene are mixed with a polystyrene solution and then spin-coated on the flat surface of a SIL to form the bulk reference sample named CNT@Matrix.

Sample characterization. In order to perform structural characterizations, part of a solution of core-shell structures is deposited on a silicon substrate. Figure S1 displays images of different core-shell structures taken with a Hitachi S-4500 Scanning Electron Microscope (SEM). It shows tubular structures compatible with laser nanotubes wrapped with a polymer layer. Likewise, atomic force microscopy (AFM) measurements has been performed using either a Veeco Dimension 3100 AFM or a Multimode AFM equipped with Nanoscope IIIa controllers. Figure 1(b) and (c) display typical AFM images of CNT@PFO/PS and CNT@PS/PS structures. The deposition of the core-shell structures leads to well separated objects making a thorough height analysis possible. Note that this is not the case when bare nanotubes are wrapped in usual surfactant. This highlights the advantage of using organic dispersions, which is made possible with the present polymer capping. Figure 1(e) and (f) show histograms of heights for both structures. In order to build these histograms, the height is measured several times along the same object and the median value is reported. In the case of CNT@PS/PS hybrids, the polymer is almost homogenously wrapped around the nanotube. The histogram is peaked and can be fitted with a Gaussian distribution centred at $\sim 3.5 \mathrm{~nm}$ for the full height of the objects. The 
(a)
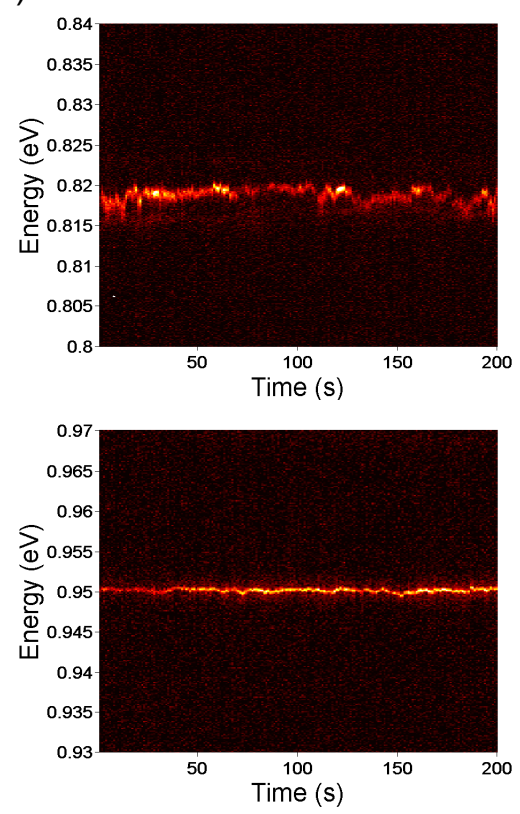

(b)
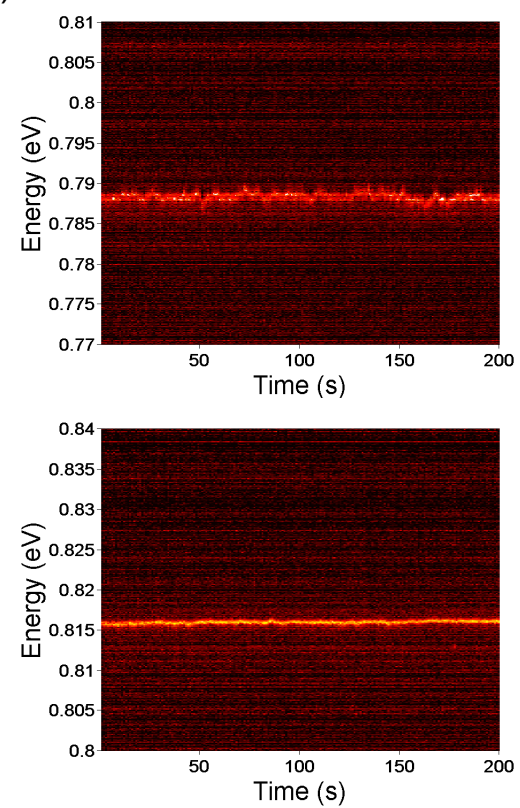

(c)
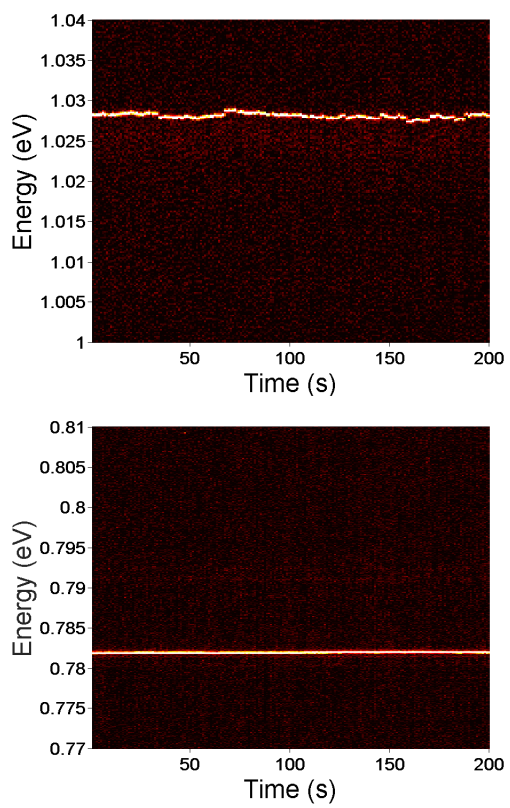

Figure 2: Temporal evolution of the spectra of single nanotubes recorded at $5 \mathrm{~K}$ with a $1 \mathrm{~s}$ integration time. (a) CNT@PFO/PS core-shell structures. (b) CNT@PS/PS core-shell structures. (c) CNT@Matrix. The top traces correspond to an example of typically unstable nanotube for each of the samples. The bottom traces correspond to typically stable ones.

histogram of CNT@PFO/PS hybrids shows a peaked distribution, centred around $6.5 \mathrm{~nm}$, together with counts above $10 \mathrm{~nm}$. These are due to the presence of big clusters of polymer attached to the nanotubes. Therefore, one can deduce an average value of the shell thickness for both structures: $1.2 \mathrm{~nm}$ for CNT@PS/PS and $\sim 2.7 \mathrm{~nm}$ for CNT@PFO/PS ones when big clusters are excluded.

Optical experiments. SIL are attached to the top of the cold finger of a cryostation from Montana Instruments. All experiments are performed at $\sim 5 \mathrm{~K}$. The nanotubes are excited at $905 \mathrm{~nm}$, approximately in resonance with their second excitonic transition $\left(\mathrm{S}_{22}\right)$, through a Mitutoyo objective $(\mathrm{NA}=0.7)$. The photoluminescence is collected with the same objective, dispersed in an Acton SP2300i (Princeton Instruments) spectrometer and detected with a nitrogen cooled InGaAs array (OMA V, Princeton Instruments). Figure 1(d) displays typical photoluminescence spectra of single core-shell structures and nanotubes in matrix. The luminescence energy is in the range of $0.8 \mathrm{eV}$ i.e. at telecommunication wavelengths.

\section{Results and Discussion}

Figure 2(a), (b) and (c) displays PL spectra as a function of time for CNT@PFO/PS, CNT@PS/PS and CNT@Matrix respectively. As an example, we display in the upper panel the typical spectrum for a nanotube considered as unstable for each of the samples. On the contrary, in the bottom panel we display our observation for a typically stable CNT. If all samples could present blinking and spectral diffusion, it seems that this effect is stronger for CNT@PFO/PS (figure 2(a)), and that CNT@PS/PS is qualitativelly more stable (figure 2(b)) and even compare well with the CNT@Matrix sample (figure 2(c)). In order to get quantitative information on the blinking phenomenon we use a Mandel analysis, calculating Mandel parameters:

$$
\mathrm{Q}=\frac{<n^{2}>-<n>^{2}}{<n>}-1
$$

where $n$ is the number of counts per time 
bins. This parameter describes the deviation of the emission statistics from a Poissonian distribution. When $\mathrm{Q} \sim 0$, the emission is shot noise limited. This parameter has been used for instance to characterize the emission stability of CdSe nanocrystals. ${ }^{25}$ When no particular care is taken to prevent blinking, these nanocrystals can show Mandel parameters Q up to $10^{5}$ in recording conditions similar to the present study. ${ }^{25}$ The CNT@PFO/PS structures show a large dispersion of Mandel parameters ranging from few unities to few hundreds. The mean value of $<Q_{P F O / P S}>$ parameter is $\sim 115$. On the contrary, the distribution of Mandel parameter of CNT@PS/PS structures is much less dispersed, with a mean value $<Q_{P S / P S}>\sim 9$. Therefore, the CNT@PS/PS structure is, in average, one order of magnitude more stable in terms of blinking than the PS/PFO structures. The complete statistics of Mandel parameters is reported on figure S4. For comparison, the luminescence of micelle wrapped CoMoCat nanotubes deposited on polylysine, reported few years ago, shows $Q$ factors of the order of few hundreds (see figure S2). This shows that the PS/PS shells indeed protect, at least partially, the nanotube from environmental fluctuations.

In the following, we investigate the emission stability in terms of spectral diffusion. The calculated standard deviation of the central energy of the emission line, calculated over $200 \mathrm{~s}$ with an integration time of $1 \mathrm{~s}$, of CNT@PFO/PS structures ranges from $\sim 90 \mu \mathrm{eV}$ to $\sim 250 \mu \mathrm{eV}$. For CNT@PS/PS structures it ranges from $\sim 60 \mu \mathrm{eV}$ to $\sim 200 \mu \mathrm{eV}$. A figure of merit for the emission stability is the ratio of the amplitude of the frequency shift versus the emitter linewidth. Here, for the best core-shell structures this figure of merit is about $1 / 10$ of their linewidth which signifies a good luminescence stability. The results of spectral stability can be compared with ones recorded for micelle wrapped CoMoCat nanotubes (see SI). In this case the standard deviation of the central energy of the emission line can be of the order of 1.7 to $2 \mathrm{meV}$, therefore, one order of magnitude larger than for both core-shell structures. This again confirms that the shell structure pro- tects the carbon nanotube. Finally, we can compare the results obtained on the core-shell structures to the ones recorded on nanotubes embedded in the polystyrene matrix. As mentioned in the introduction, this configuration has been studied before and used in CQED experiments thanks to the emission stability of nanotubes. ${ }^{1}$ Therefore, this represents a relevant control sample. In optimal fabrication conditions (see SI), the nanotubes emission is very stable both for blinking and spectral diffusion (see figure 2(c)). Indeed, the mean value of the Mandel parameter measured on this sample is $\left\langle Q_{\text {matrix }}>\sim 14\right.$ which is quite comparable with what we obtained on the CNT@PS/PS core-shell structure. This comparison demonstrates that the core-shell approach is as efficient as the inclusion of nanotubes in a matrix to prevent blinking, while keeping the possibility of handling and processing the objects. In addition, the nanotubes embedded in the PS matrix show very limited spectral diffusion. Indeed, the calculated standard deviation is typically of the order of few tenth of $\mu \mathrm{eV}$ for an emission linewidth which is of the order of few hundreds of $\mu \mathrm{eV}$. Here the mean value of the standard deviation is $\sim 30 \mu \mathrm{eV}$. The comparison shows that the best values of the core-shell structures reach the one of the nanotubes in the matrix. This demonstrates again the suitability of the core-shell approach.

In order to go further in the quantitative study of the spectral diffusion, we performed a Allan deviation analysis. This method is commonly used to investigate the frequency stability of oscillators over time ${ }^{26}$ and has been recently applied to carbon nanotubes. ${ }^{16}$ One of the advantages of this analysis method is to infer the type of noise. In fact, the slope of the Allan deviation depends on the origin of the noise. For instance, a frequency drift leads to a $\tau^{+1}$ slope whereas a flicker noise leads to a $\tau^{0}$ slope. The Allan deviation is defined as:

$$
\begin{array}{r}
\sigma_{\mathrm{e}}^{2}(\tau)=\frac{1}{2}<\left(\overline{e_{k+1}}-\overline{e_{k}}\right)^{2}>= \\
\frac{1}{2} \lim _{N \rightarrow \infty}\left[\frac{1}{N} \sum_{k=1}^{N}\left(\overline{e_{k+1}}-\overline{e_{k}}\right)^{2}\right]
\end{array}
$$




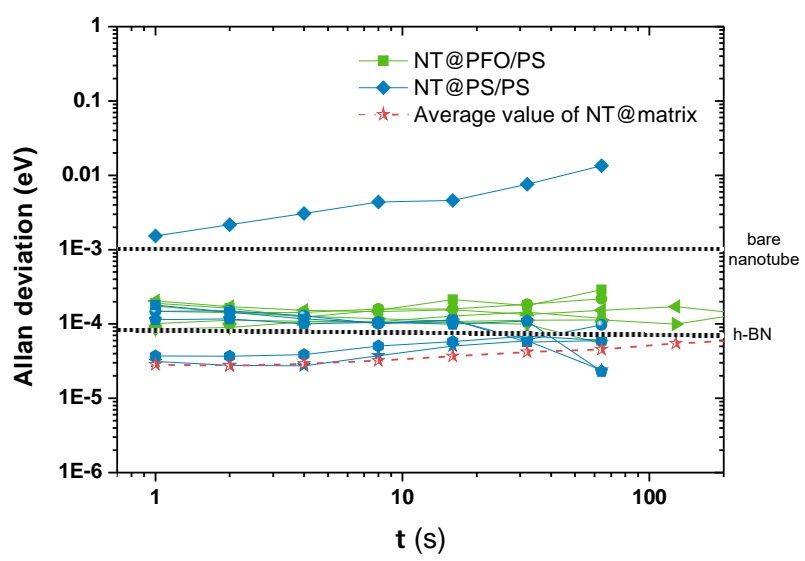

Figure 3: Allan deviation of CNT@PS/PS (blue), CNT@PFO/PS (green) and mean value of CNT@Matrix (red). All the Allan curves for CNT@Matrix are depicted on figure S5. The dotted black lines are guide for the eye showing values of Allan deviation for CoMoCat CNT on $\mathrm{SiO}_{2}$ and h-BN extracted from ref. ${ }^{16}$

$$
\overline{\mathrm{e}_{\mathrm{k}}}=\frac{1}{\tau} \int_{t_{k}}^{t_{k+1}} e(t) \mathrm{d} t
$$

where $\overline{e_{k}}$ is the mean value of the energy $e(t)$ of the line on the time interval $\tau$.

Figure 3 displays the Allan deviation as a function of the interval $\tau$ for six CNT@PFO/PS (green curves) and six CNT@PS/PS (blue curves) structures. The red stars in figure 3 are the average values of the Allan deviations of nanotubes in matrix. The whole statistics of CNT@Matrix is available in SI (figure S5). Except for one CNT@PS/PS, all the core-shell structures have their Allan deviation between few $10^{-5}$ few $10^{-4} \mathrm{eV}$, slightly above the average value of CNT in matrix. For comparison purpose, Figure S6 shows several time traces and their Allan deviation curves for different degree of stability. Moreover, the variation of the Allan deviation with time is relatively flat. Therefore, we can assume that the Allan deviation in both CNT@PFO/PS and CNT@PS/PS shows a close to $\tau^{0}$ variation. In this framework, this would mean that spectral diffusion of core-shell structures is a flicker noise. In electronics this type of noise can be observed, for instance, when charges are randomly emitted by impurities. If one assumes that charges in the environment of the nanotubes are trapped and can jump randomly from one trap to another, the random variation of the charge distribution to nanotube distance would account for this type of noise of the luminescence frequency due to Stark effect. Finally, the Allan deviation analysis allows to compare the performances of our core-shell structures to other configurations reported in the literature. In fact, Noé et al recently reported the same analysis for bare nanotubes on a $\mathrm{SiO}_{2}$ surface and on a h-BN substrate (see guides for the eye in figure 3). ${ }^{16}$ First, the Allan deviations measured for our core-shell structures are one or two orders of magnitude lower than the one reported for a nanotube on a $\mathrm{SiO}_{2}$ surface that reached $\sim 10^{-3}$ in average. This comparison is in agreement with what we measured for CoMoCat nanotubes on polylysine (see SI). Moreover it strongly supports the fact that the double shell structure is indeed protecting, at least partially, the nanotube from the charge fluctuations of the substrate. Moreover, the Allan deviations of the core-shell structures displayed on figure 3 are of the same order of magnitude (few $10^{-5}$ to few $10^{-4}$ ) as the one reported for a nanotube on the h-BN substrate (few $10^{-5}$ ), which is considered as a material of choice to stabilize quantum emitters luminescence. This comparison shows that the core-shell structures can reach the best performance reported in the literature, while offering the possibility to be handle and to process the single emitters on any substrate of interest.

\section{Conclusion}

In conclusion, we report on a quantitative study of blinking and spectral diffusion processes of core-double shell structures based on single carbon nanotubes emitting at the telecommunication wavelength. The double shell structure is shown to protect the nanotube from the fluctuations of its local environment leading to a strong reduction of both blinking and spectral diffusion. The quantitative comparison with litera- 
ture highlights that these structures can reach the performance of single nanotubes laid on $h$ BN while being easy to handle and to process on any substrate of interest. These results show that the core-shell approach is relevant in the context of the use of single-walled carbon nanotubes in applications needing quantum emitters at telecommunication wavelengths.

\section{Supporting Information Available:} This material is available free of charge via the Internet at http://pubs.acs.org/.

Acknowledgement The authors are grateful to A. Högele for sharing raw data of single carbon nanotubes on $\mathrm{SiO}_{2}$ and h-BN. J.S. Lauret is member of "Institut Universitaire de France".

\section{References}

(1) Jeantet, A.; Chassagneux, Y.; Raynaud, C.; Roussignol, P.; Lauret, J. S.; Besga, B.; Estève, J.; Reichel, J.; Voisin, C. Widely Tunable Single-Photon Source from a Carbon Nanotube in the Purcell Regime. Physical Review Letters 2016, 116, 247402.

(2) Jeantet, A.; Chassagneux, Y.; Claude, T.; Roussignol, P.; Lauret, J. S.; Reichel, J.; Voisin, C. Exploiting One-Dimensional Exciton-Phonon Coupling for Tunable and Efficient Single-Photon Generation with a Carbon Nanotube. NanoLetters 2017, 17, 4184.

(3) Imamura, S.; Watahiki, R.; Miura, R.; Shimada, T.; Kato, Y. K. Optical control of individual carbon nanotube light emitters by spectral double resonance in silicon microdisk resonators. Applied Physics Letters 2013, 102, 161102.

(4) Khasminskaya, S.; Pyatkov, F.; Karolina, S.; Ferrari, S.; Kahl, O.; Kovalyuk, V.; Rath, P.; Vetter, A.; Hennrich, F.; Kappes, M. M.; Gol, G.; Korneev, A.; Rockstuhl, C.; Krupke, R.; Pernice, W. H. P. Fully integrated quantum photonic circuit with an electrically driven light source. Nature Photonics 2016, 10, 727-732.

(5) He, X.; Htoon, H.; Doorn, S. K.; Pernice, W. H. P.; Pyatkov, F.; Krupke, R.; Jeantet, A.; Chassagneux, Y.; Voisin, C. Carbon nanotubes as emerging quantumlight sources. Nature Materials 2018, 17, 663-670.

(6) Luo, Y.; Ahmadi, E. D.; Shayan, K.; Ma, Y.; Mistry, K. S.; Zhang, C.; Hone, J.; Blackburn, J. L.; Strauf, S. Purcell-enhanced quantum yield from carbon nanotube excitons coupled to plasmonic nanocavities. Nature Communications 2017, 8, 1413.

(7) Uda, T.; Ishii, A.; Kato, Y. K. Single Carbon Nanotubes as Ultrasmall All-Optical Memories. ACS Photonics 2018, 5, 559.

(8) Yoshida, M.; Kumamoto, Y.; Ishii, a.; Yokoyama, a.; Kato, Y. K. Stark effect of excitons in individual air-suspended carbon nanotubes. Applied Physics Letters 2014, 105, 161104.

(9) Gaufrès, E.; Izard, N.; Le Roux, X.; Kazaoui, S.; Marris-Morini, D.; Cassan, E.; Vivien, L. Optical microcavity with semiconducting single-wall carbon nanotubes. Optics Express 2010, 18, 5740-5.

(10) Legrand, D.; Roquelet, C.; Lanty, G.; Roussignol, P.; Lafosse, X.; Bouchoule, S.; Deleporte, E.; Voisin, C.; Lauret, J. S. Monolithic microcavity with carbon nanotubes as active material. Applied Physics Letters 2013, 102, 153102.

(11) He, X.; Hartmann, N. F.; Ma, X.; Kim, Y.; Ihly, R.; Blackburn, J. L.; Gao, W.; Kono, J.; Yomogida, Y.; Hirano, A.; Tanaka, T.; Kataura, H.; Htoon, H.; Doorn, S. K. Tunable room-temperature single-photon emission at telecom wavelengths from sp3 defects in carbon nanotubes. Nature Photonics 2017, 11, 577. 
(12) Luo, Y.; He, X.; Kim, Y.; Blackburn, J. L.; Doorn, S. K.; Htoon, H.; Strauf, S. Carbon Nanotube Color Centers in Plasmonic Nanocavities: A Path to Photon Indistinguishability at Telecom Bands. Nano Letters 2019, 19, 9037.

(13) Battie, Y.; Ducloux, O.; Thobois, P.; Dorval, N.; Lauret, J.; Attal-Trétout, B.; Loiseau, A. Gas sensors based on thick films of semi-conducting single walled carbon nanotubes. Carbon 2011, 49, 3544.

(14) Ai, N.; Walden-newman, W.; Song, Q.; Kalliakos, S.; Strauf, S. Suppression of Blinking and Enhanced Exciton Emission from Individual Carbon Nanotubes. ACS Nano 2011, 2664-2670.

(15) Walden-newman, W.; Sarpkaya, I.; Strauf, S. Quantum Light Signatures and Nanosecond Spectral Diffusion from Cavity-Embedded Carbon Nanotubes. Nanoletters 2012, 12, 1934.

(16) Noé, J. C.; Nutz, M.; Reschauer, J.; Morell, N.; Tsioutsios, I.; ReserbatPlantey, A.; Watanabe, K.; Taniguchi, T.; Bachtold, A.; Högele, A. Environmental Electrometry with Luminescent Carbon Nanotubes. NanoLetters 2018, 18, 41364140 .

(17) Chen, O.; Zhao, J.; Chauhan, V. P.; Cui, J.; Wong, C.; Harris, D. K.; Wei, H.; Han, H.-s.; Fukumura, D.; Jain, R. K.; Bawendi, M. G. Compact high-quality CdSe-CdS core-shell nanocrystals with narrow emission linewidths and suppressed blinking. Nature Materials 2013, 12, 445-451.

(18) Ji, B.; Giovanelli, E.; Habert, B.; Spinicelli, P.; Nasilowski, M.; Xu, X.; Lequeux, N.; Hugonin, J.-p.; Marquier, F.; Greffet, J.-j.; Dubertret, B. Non-blinking quantum dot with a plasmonic nanoshell resonator. Nature Nanotechnology 2015, $1-6$.
(19) Orcin-Chaix, L.; Trippé-Allard, G.; Voisin, C.; Okuno, H.; Derycke, V.; Lauret, J.-S.; Campidelli, S. Single-Walled Carbon Nanotube/Polystyrene Core-Shell Hybrids: Synthesis and Photoluminescence Properties. Journal of Material Chemistry $C$ 2018, 6, 4786.

(20) Lauret, J.-s.; Voisin, C.; Cassabois, G.; Delalande, C.; Roussignol, P.; Jost, O.; Filoramo, a.; Capes, L. Bandgap Photoluminescence of Semiconducting Single Wall Carbon Nanotubes. Physica E 2004, 21, 1057.

(21) Nguyen, D. T.; Voisin, C.; Roussignol, P.; Roquelet, C.; Lauret, J. S.; Cassabois, G. Elastic Exciton-Exciton Scattering in Photoexcited Carbon Nanotubes. Physical Review Letters 2011, 10\%, 127401.

(22) Graf, A.; Zakharko, Y.; Schießsl, S. P.; Backes, C.; Pfohl, M.; Flavel, B. S.; Zaumseil, J. Large scale, selective dispersion of long single-walled carbon nanotubes with high photoluminescence quantum yield by shear force mixing. Carbon 2016, 105, 593-599.

(23) Graf, A.; Held, M.; Zakharko, Y.; Tropf, L.; Gather, M. C.; Zaumseil, J. Electrical pumping and tuning of excitonpolaritons in carbon nanotube microcavities. Nature Materials 2017, 16, 911-918.

(24) Jeantet, A.; Chassagneux, Y.; Claude, T.; Lauret, J. S.; Voisin, C. Interplay of spectral diffusion and phonon-broadening in individual photo-emitters: the case of carbon nanotubes. Nanoscale 2018, 10, 683689 .

(25) Margolin, G.; Protasenko, V.; Kuno, M.; Barkai, E. Photon Counting Statistics for Blinking CdSe-ZnS Quantum Dots: A Lévy Walk Process. The Journal of Physical Chemistry B 2006, 110, 19053-19060, PMID: 16986903. 
(26) Kozlova, O. Caractérisation d'une horloge à piégeage cohérent de population dans une vapeur thermique de césium. Principaux effets pouvant affecter la stabilité de fréquence á moyen-long terme. Physique Atomique [physics.atomph]. Université Pierre et Marie Curie Paris VI. 2012, $00669784 f$. 
Table 1: Summary of average values of Mandel parameters for the different type of samples

\begin{tabular}{|c|c|c|c|c|}
\hline & CNT@PFO/PS & CNT@PS/PS & CoMoCat@SiO & CNT@Matrix \\
\hline$\langle Q\rangle$ & 115 & 9 & few hundreds & 14 \\
\hline
\end{tabular}


Graphical TOC Entry

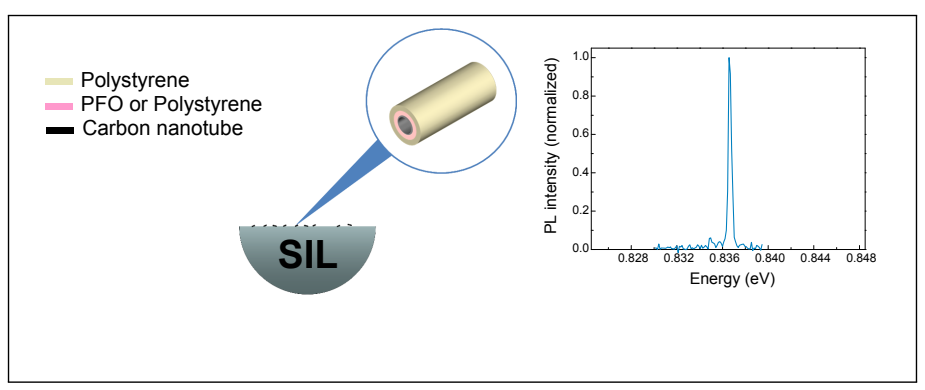

